\title{
Cambios tempranos de la superficie corneal posterior a cirugía refractiva evaluados con un analizador de superficie ocular
}

\section{Early corneal surface changes after refractive surgery evaluated with an ocular surface analyzer}

\author{
Carlos Adolfo Müller-Morales, ${ }^{*}$ Naomi Zatarain-Barrón,, \\ Enrique Octavio Graue-Hernández, ${ }^{*}$ Alejandro Navas-Pérez*
}

Citar como: Müller-Morales CA, Zatarain-Barrón N, Graue-Hernández EO, Navas-Pérez A. Cambios tempranos de la superficie corneal posterior a cirugía refractiva evaluados con un analizador de superficie ocular. An Med (Mex). 2020 ; 65 (4): $270-274$.

https://dx.doi.org/10.35366/97464

\section{RESUMEN}

Introducción: Existe controversia respecto al papel que desempeña la cirugía refractiva en el desarrollo posterior de resequedad ocular. Objetivo: Evaluar los cambios tempranos en la superficie corneal después de cirugía refractiva tipo laser assisted in situ keratomileusis (LASIK por sus siglas en inglés) con un analizador de superficie ocular. Material y métodos: Se realizaron mediciones preoperatorias y una semana posterior al LASIK se evaluó la puntuación en escala de ocular surface disease index (OSDI por sus siglas en inglés), tiempo de ruptura lagrimal no invasivo (segundos), tamaño de menisco lagrimal $(\mathrm{mm})$ y análisis cuantitativo de las glándulas de Meibomio (\%) con el analizador de superficie ocular LacryDiag (Quantel, Cournon d'Auvergne, Francia). Resultados: Se incluyeron 14 pacientes y 28 ojos en el estudio. Hallazgos prequirúrgicos y postquirúrgicos: puntación OSDI de

\section{ABSTRACT}

Introduction: To this day, controversy remains regarding the role refractive surgery plays in posterior development of dry eye signs and symptoms. Objective: To evaluate early changes in corneal surface after refractive surgery by laser assisted in situ keratomileusis, with an ocular surface analyzer. Material and methods: Pre and 1-week post-operative measurements of ocular surface disease index score, non-invasive tear breakup time, tear meniscus height ( $\mathrm{mm}$ ), and meibomian gland dropout (\%) were made with the ocular surface analyzer LacryDiag (Quantel, Cournon d'Auvergne, France). Results: 28 eyes of 14 patients were included. Pre-and post-op findings were as following: ocular surface disease score from 8 to $18(p=1.25)$; non-invasive tear breakup time from 10.7 seconds to 6.2 seconds right eyes and 10.9 seconds to 6.3 seconds left eye $(p=1.10)$; tear meniscus height from $0.5 \mathrm{~mm}$ both eyes to $0.3 \mathrm{~mm}$ right eye $(p=$

\footnotetext{
* Médico Especialista en Oftalmología-Córnea y Cirugía Refractiva.

‡ Médico pasante de Servicio Social.
}

Departamento de Córnea, Instituto de Oftalmología Fundación de Asistencia Privada Conde de Valenciana, IAP. Ciudad de México, México.

Recibido para publicación: 03/11/2020. Aceptado: 10/11/2020.

Correspondencia: Carlos Adolfo Müller-Morales Chimalpopoca Núm. 14, Col. Obrera, 06800, Cuauhtémoc, Ciudad de México, CDMX. Teléfono: 55 5442-1700 E-mail: mumolfo@gmail.com

$$
\begin{aligned}
& \text { Abreviaturas: } \\
& \mathrm{D}=\text { Dioptrías. } \\
& \text { LASIK }=\text { Laser assisted in situ keratomileusis. } \\
& P R K=\text { Photorefractive keratectomy. } \\
& \text { SMILE }=\text { Small Incision Lenticule Extraction. } \\
& \text { OD }=\text { Ojo derecho. } \\
& \text { OI }=\text { Ojo izquierdo. } \\
& \text { OSDI }=\text { Ocular surface disease index. } \\
& \text { OU }=\text { Ambos ojos. }
\end{aligned}
$$


8 a $18(\mathrm{p}=1.25)$; tiempo de ruptura lagrimal no invasivo de 10.7 segundos a 6.2 segundos en ojo derecho y de 10.9 segundos a 6.3 segundos en ojo izquierdo $(\mathrm{p}=1.10)$; altura de menisco lagrimal de $0.5 \mathrm{~mm}$ en ambos ojos a $0.3 \mathrm{~mm}$ en ojo derecho $(\mathrm{p}=$ 1.64) y $0.2 \mathrm{~mm}$ en ojo izquierdo (1.64); y porcentaje de pérdida de glándulas de Meibomio de 21.6 a $21 \%$ en ojo derecho y de 23.6 a $23 \%$ en ojo izquierdo $(p=1.50)$. Conclusiones: Aunque no se encontraron diferencias estadísticamente significativas entre valores prequirúrgicos y postquirúrgicos, sí existieron diferencias clínicas y sintomáticas en los pacientes.

Palabras clave: Ojo seco, cirugía refractiva, LASIK.

Nivel de evidencia: IV

\section{INTRODUCCIÓN}

El ojo seco posterior a cirugía refractiva es un problema común al que se enfrenta el oftalmólogo. Los síntomas de resequedad ocular después de cirugía refractiva son de magnitud variable y producen insatisfacción en el paciente, así como frustración por parte del cirujano. En algunos reportes se ha observado que hasta $20 \%$ de pacientes operados de cirugía refractiva son derivados con el corneólogo debido a la falta de satisfacción postratamiento. ${ }^{1} \mathrm{El}$ incremento en el tiempo invertido en el tratamiento refractivo, la discrepancia ocasional entre síntomas manifestados por el paciente en comparación con los signos clínicos observados, y la técnica quirúrgica a elegir de acuerdo con las características individuales de cada paciente (laser assisted in situ keratomileusis [LASIK, por sus siglas en inglés], PRK [Photorefractive keratectomy], trans-PRK, SMILE [Small Incision Lenticule Extraction]) son sólo algunos de los retos que desafían al cirujano refractivo. ${ }^{2,3}$

Es conocido que el ojo seco preexistente a la cirugía refractiva es un factor de riesgo importante de desarrollar ojo seco postoperatorio grave. ${ }^{4,5} \mathrm{En}$ algunos reportes se ha estimado la existencia de signos y síntomas de ojo seco previo a cirugía refractiva hasta en 50 y $30 \%$ de los casos, respectivamente. ${ }^{6}$ Otros factores de riesgo incluyen mayor corrección refractiva (-9D a -14D), mayor tiempo de succión, diámetro y profundidad de la zona de ablación, grosor del flap, raza asiática, sexo femenino, uso crónico de lentes de contacto, polimorfismos en el gen THBS1, y cirugías oculares previas como blefaroplastia y corrección de lagoftalmos. ${ }^{7-12}$

Los mecanismos fisiopatológicos por los cuales se produce ojo seco posterior al LASIK aún no son del todo conocidos. Lo más aceptado hoy en día es una etiología multifactorial, cuyos componentes incluyen disminución en la influencia trófica en el epitelio cor-
1.64) and $0.2 \mathrm{~mm}$ left eye (1.64); and meibomian gland dropout from $21.6 \%$ to $21 \%$ right eye and $23.6 \%$ to $23 \%$ left eye ( $p=1.50$ ). Conclusions: Although statistically significant differences were not found between pre-and post-op measurements, there were clinical and symptomatic differences in patients.

Keywords: Dry eye, refractive surgery, LASIK.

Level of evidence: $I V$

neal secundario a amputación de los nervios corneales, reducción de la sensibilidad corneal y el reflejo de parpadeo, daño a las células caliciformes limbales producido por la presión del anillo de succión, alteración de la estabilidad lagrimal por los cambios en la curvatura de la córnea, la inflamación propia del evento quirúrgico y el efecto tóxico del tratamiento tópico. ${ }^{13}$

La anamnesis del paciente es el factor más importante en la evaluación del ojo seco posterior al LASIK, puesto que no hay una sola prueba que sea suficiente para su diagnóstico y los signos y síntomas en ocasiones no se correlacionan. Asimismo, se ha visto que los síntomas postoperatorios del ojo seco son de naturaleza temporal, más pronunciados durante el primer mes y generalmente duran menos de 12 meses después de la cirugía. ${ }^{14}$

En cuanto a hallazgos objetivos de resequedad ocular, la duración de éstos posterior a cirugía refractiva puede ser variable y dependiente de distintos factores. En el estudio de Nassaralla y colaboradores se evaluaron los cambios en la sensibilidad corneal después del LASIK para la corrección de diferentes grados de miopía, encontrando que la sensibilidad de la córnea disminuye en las áreas central y paracentral hasta por nueve meses, siendo la profundidad de la ablación un factor importante en la disminución temporal de la sensibilidad corneal y su recuperación. ${ }^{15} \mathrm{Hu}$ y su equipo investigaron la relación entre los meniscos lagrimales y la densidad del nervio corneal en pacientes después del LASIK, y concluyeron que los meniscos lagrimales y nervios corneales periféricos se recuperaron continuamente después del LASIK durante la etapa temprana; sin embargo, el volumen de lágrima puede depender de los nervios corneales centrales residuales al mes en lugar de la recuperación del nervio corneal. ${ }^{16}$ En un estudio conducido por Tao y colaboradores ${ }^{17}$ se determinaron los meniscos de lágrimas superiores e inferiores 
mediante tomografía de coherencia óptica (OCT) en voluntarios después del LASIK para tratar miopía, encontrando que tanto el menisco lagrimal superior como el inferior disminuyen y se recuperan gradualmente hasta 20 meses después del LASIK.

A pesar de todo esto, aun si hay controversia respecto al papel que desempeña la cirugía refractiva en el desarrollo posterior de sequedad ocular, existen reportes a favor de este planteamiento, en los que se han descrito resultados en los cuales $94.8,85.4$ y $59.4 \%$ de pacientes experimentaron síntomas de ojo seco a un día, una semana y un mes después de la cirugía, respectivamente. ${ }^{13}$ También se han constatado estudios con resultados contrarios que señalan que el ojo seco después del LASIK es poco frecuente. ${ }^{18}$ Esta variabilidad en los hallazgos es atribuible a la existencia de pocos estudios prospectivos controlados, al corto tiempo de seguimiento postoperatorio de pacientes y a la falta de estandarización de criterios de evaluación para ojo seco.

El objetivo primario de nuestro estudio fue analizar los cambios tempranos en la superficie ocular posterior a cirugía refractiva tipo LASIK con un analizador de superficie ocular. El objetivo secundario fue determinar el grado de severidad de sintomatología manifestada por el paciente a través de la escala OSDI.

\section{MATERIAL Y MÉTODOS}

El diseño de esta investigación consiste en un estudio de intervención, no aleatorizado, prospectivo, comparativo, longitudinal. Se incluyeron pacientes mayores de 18 años, sin patología o tratamiento ocular preexistente, que firmaran el consentimiento informado y que cumplieran con las fechas de seguimiento y los cuidados postoperatorios indicados. Se excluyeron de este estudio los casos que no cumplieron con estos criterios. Se incluyó un total de 28 ojos y 14 pacientes para someterse a cirugía refractiva tipo LASIK en ambos ojos en el periodo de marzo a junio de 2019. Previo a la cirugía, se registraron datos demográficos de los pacientes, así como mediciones basales de las variables desenlace: puntuación en escala de OSDI, tiempo de ruptura lagrimal no invasivo (NIBUT) (segundos), tamaño de menisco lagrimal $(\mathrm{mm})$ y análisis cuantitativo de las glándulas de Meibomio (\%) con el analizador de superficie ocular LacryDiag (Quantel, Cournon d'Auvergne, France). A todos los pacientes se les realizó cirugía refractiva tipo LASIK. Según los requerimientos del paciente por sus queratometrías y paquimetrías, se utilizó el microqueratomo Moria M2SU (MORIA
SA, France). Posterior a la creación y levantamiento del flap, se realizó aplicación del Excimer láser (Schwind Amaris) tras un adecuado centrado de las miras del láser y la correcta activación del EyeTracker, indicando al paciente mantener su mirada fija en un estímulo luminoso. Posteriormente, se eliminaron los detritos presentes sobre el estroma corneal expuesto con microesponjas húmedas. Por último, se realizó el reposicionamiento del colgajo sobre el estroma corneal. Como parte del tratamiento tópico postoperatorio se aplicó una gota de antibiótico tópico (Vigamoxi-Alcon Laboratories, Inc. Fort Worth, Texas-United States) cada seis horas, hialuronato de sodio $0.4 \%$ (Lagricel-Laboratorios Sophia. Guadalajara, Jalisco, México) y esteroide acetato de prednisolona al 1\% (Prednefrin-Alcon Laboratories, Inc. Fort Worth, Texas-United States).

Las mediciones de las variables de desenlace se realizaron nuevamente una semana postratamiento. Para el análisis estadístico de los resultados se utilizó una prueba de T-pareada. Un valor de $\mathrm{p}<0.05$ se consideró estadísticamente significativo.

\section{RESULTADOS}

En el estudio se incluyó un total de 14 pacientes y 28 ojos, se sometieron a cirugía refractiva tipo LASIK siete $(50 \%)$ hombres y siete $(50 \%)$ mujeres. La edad promedio fue de 24.7 años (rango de 21-31 años).

El diagnóstico refractivo más frecuente fue astigmatismo miópico compuesto, encontrado en 23 (82\%) de los ojos evaluados. La refracción esférica promedio de ojo derecho fue de $-2.7 \mathrm{D}$, con un rango de -0.5 a $-5.5 \mathrm{D}$, y un cilindro promedio de $-1.6 \mathrm{D}$, con un rango

Tabla 1: Características demográficas de la población en estudio $(\mathrm{N}=14)$.

\begin{tabular}{ll}
\hline Característica & $\mathrm{n}(\%)$ \\
\hline Masculino & $7(50)$ \\
Femenino & $7(50)$ \\
Edad promedio & 24.7 \\
Astigmatismo miópico compuesto & $23(82)$ \\
Refracción esférica promedio & \\
$\quad$ Ojo derecho & $-2.7 \mathrm{D}$ \\
$\quad$ Ojo izquierdo & $-2.7 \mathrm{D}$ \\
Cilindro promedio & \\
$\quad$ Ojo derecho & $-1.6 \mathrm{D}$ \\
$\quad$ Ojo izquierdo & $-1.4 \mathrm{D}$ \\
Equivalente esférico promedio & \\
$\quad$ Ojo derecho & $-3.5 \mathrm{D}$ \\
$\quad$ Ojo izquierdo & $-3.4 \mathrm{D}$ \\
\hline
\end{tabular}


Tabla 2: Resultados prequirúrgicos y postquirúrgicos.

\begin{tabular}{|c|c|c|c|c|c|}
\hline \multirow[b]{2}{*}{ Variable } & \multicolumn{2}{|c|}{ Pre-LASIK } & \multicolumn{2}{|c|}{ Post-LASIK } & \multirow[b]{2}{*}{$p$} \\
\hline & OD & $\mathrm{Ol}$ & OD & Ol & \\
\hline OSDI & \multicolumn{2}{|c|}{8} & \multicolumn{2}{|c|}{18} & 1.25 \\
\hline NIBUT (s) & 10.7 & 10.9 & 6.2 & 6.3 & 1.10 \\
\hline Menisco lagrimal $(\mathrm{mm})$ & 0.5 & 0.5 & 0.3 & 0.2 & 1.64 \\
\hline $\begin{array}{l}\text { Pérdida de glándulas } \\
\text { de Meibomio }(\%)\end{array}$ & 21.6 & 23.6 & 21.0 & 23.0 & 1.50 \\
\hline
\end{tabular}

de 0 a -5D. La refracción esférica promedio de ojo izquierdo fue de $-2.7 \mathrm{D}$, con un rango de -0.5 a $-5.00 \mathrm{D}$, y un cilindro promedio de $-1.4 \mathrm{D}$, con un rango de 0 a $-4.00 \mathrm{D}$. El equivalente esférico promedio en ojo derecho fue de -3.5D y en ojo izquierdo de -3.4D (Tabla 1).

Los hallazgos promedio de las variables medidas previo a cirugía consistieron en: puntuación en escala de OSDI de 8; NIBUT de 10.7 segundos en ojo derecho y 10.9 segundos en ojo izquierdo; altura de menisco lagrimal de $0.5 \mathrm{~mm}$ en ambos ojos; y porcentaje de pérdida de glándulas de Meibomio de 21.6\% en ojo derecho y $23.6 \%$ en ojo izquierdo.

La evaluación postquirúrgica a una semana mostró los siguientes hallazgos promedio: puntación en escala de OSDI de 18 ( $\mathrm{p}=1.25)$; NIBUT de 6.2 segundos en ojo derecho y 6.3 segundos en ojo izquierdo $(\mathrm{p}=1.10)$; altura de menisco lagrimal de $0.3 \mathrm{~mm}$ en ojo derecho $(\mathrm{p}=1.64)$ y $0.2 \mathrm{~mm}$ en ojo izquierdo (1.64); y porcentaje de pérdida de glándulas de Meibomio de $21 \%$ en ojo derecho y $23 \%$ en ojo izquierdo $(\mathrm{p}=1.50)($ Tabla 2 y Figura 1$)$.

\section{DISCUSIÓN}

En nuestro estudio se evaluó un total de 28 ojos con astigmatismo miópico compuesto. El error esférico entre pacientes osciló entre miopía baja-moderada (0-6D), mientras que el cilindro osciló entre astigmatismo bajo-alto $(0 \geq 3 \mathrm{D})$.

A pesar de que no se demostró significancia estadística en los resultados pre-LASIK y post-LASIK en la puntuación por escala de OSDI, sí encontramos una diferencia sintomática entre los pacientes, dado que el valor promedio prequirúrgico de 8 representa una calificación normal, en cambio el valor promedio postquirúrgico de 18 corresponde a un ojo seco de grado leve.

Utilizar un analizador de superficie ocular nos permite reconocer con mayor facilidad si existe correlación objetiva entre los cambios en la superfi-
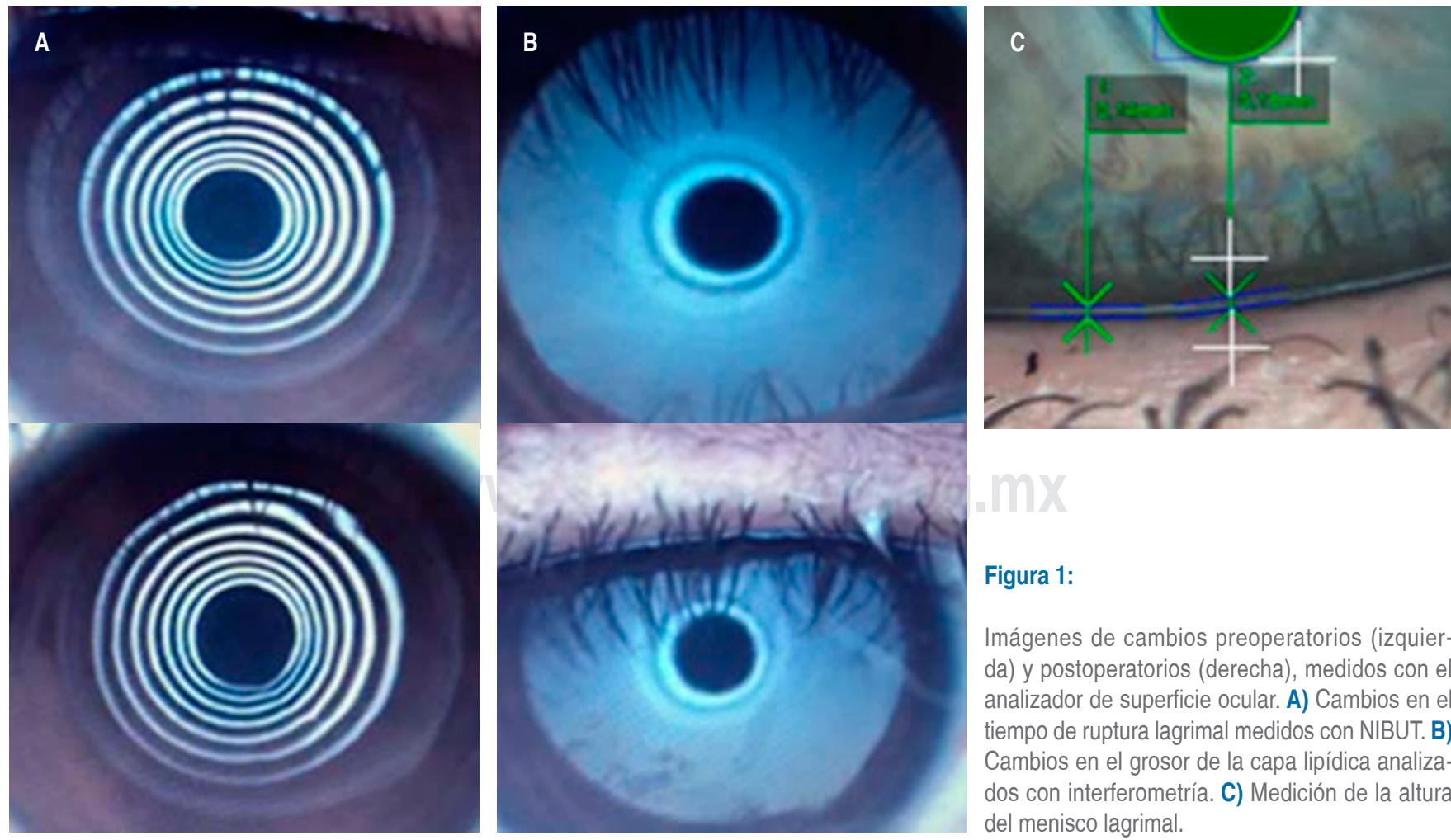

Figura 1:

Imágenes de cambios preoperatorios (izquierda) y postoperatorios (derecha), medidos con el analizador de superficie ocular. A) Cambios en el tiempo de ruptura lagrimal medidos con NIBUT. B) Cambios en el grosor de la capa lipídica analizados con interferometría. C) Medición de la altura del menisco lagrimal. 
An Med (Mex) 2020; 65 (4): 270-274

cie, con respecto a los cambios subjetivos observados por la escala de OSDI. Al observar los hallazgos pre-LASIK y post-LASIK en NIBUT observamos de nuevo que el cambio no fue estadísticamente significativo; sin embargo, la diferencia promedio de cambio fue de 4.2 segundos. La reducción en NIBUT ha sido reportada previamente como uno de los primeros cambios posteriores a cirugía refractiva que puede perdurar por lapsos de hasta un mes, ${ }^{19}$ el cual es un dato que nuestro estudio permite apoyar.

La altura de menisco lagrimal normal es de 0.2$0.5 \mathrm{~mm}$ en la población general con ojos sanos. Observamos que nuestros pacientes presentaron valores normales pre-LASIK, los cuales disminuyeron en promedio $0.2-0.3 \mathrm{~mm}$ en ambos ojos una semana postratamiento, sin significancia estadística. Esto se reconoce como un hallazgo normal debido a la disminución de producción lagrimal que existe posterior a cirugía refractiva, es secundaria al trauma nervioso inducido al momento de la creación del flap. ${ }^{16}$

La evaluación de ojo seco posterior al LASIK continúa siendo un reto para oftalmólogos. Representa una de las principales quejas entre pacientes posterior a la cirugía, y puede variar desde molestias leves y temporales hasta síntomas severos y perdurables. ${ }^{7}$ La contribución de ciertos factores de riesgo para desarrollar ojo seco después de cirugía refractiva, tales como síndrome de ojo seco previo, correcciones refractivas altas y uso de lentes de contacto, ha sido bien documentada. ${ }^{13}$

En los últimos años se han desarrollado nuevas herramientas para cuantificar los cambios de superficie ocular tanto en ojos sanos como enfermos. Un analizador de superficie ocular, como el empleado en nuestro estudio, permite abrir paso a futuras investigaciones sobre ojo seco en el contexto de cirugía refractiva.

\section{CONCLUSIÓN}

Aunque no se encontraron diferencias significativas entre valores prequirúrgicos y postquirúrgicos, sí existieron diferencias clínicas y sintomáticas entre los pacientes; un aumento en puntuación de OSDI de 8 (categoría normal) a 18 (categoría de ojo seco grado leve); una disminución de NIBUT de 4.46 segundos y una reducción de la altura de menisco lagrimal de 0.2-0.3 $\mathrm{mm}$. Aún se requieren estudios más amplios que utilicen analizadores de superficie ocular en cirugía refractiva a futuro.

\section{BIBLIOGRAFÍA}

1. Levinson BA, Rapuano CJ, Cohen EJ, Hammersmith KM, Ayres BD, Laibson PR. Referrals to the Wills Eye Institute Cornea Service after laser in situ keratomileusis: reasons for patient dissatisfaction. J Cataract Refract Surg. 2008; 34 (1): 32-39.

2. Dooley I, D’Arcy F, O'Keefe M. Comparison of dry-eye disease severity after laser in situ keratomileusis and laser-assisted subepithelial keratectomy. J Cataract Refract Surg. 2012; 38 (6): 1058-1064.

3. Nettune GR, Pflugfelder SC. Post-LASIK tear dysfunction and dysesthesia. Ocul Surf. 2010; 8 (3): 135-145.

4. Toda I, Asano-Kato N, Hori-Komai Y, Tsubota K. Laserassisted in situ keratomileusis for patients with dry eye. Arch Ophthalmol. 2002; 120 (8): 1024-1028.

5. Ambrósio R Jr, Tervo T, Wilson SE. LASIK-associated dry eye and neurotrophic epitheliopathy: pathophysiology and strategies for prevention and treatment. J Refract Surg. 2008; 24 (4): 396-407.

6. Yu EY, Leung A, Rao S, Lam DS. Effect of laser in situ keratomileusis on tear stability. Ophthalmology. 2000; 107 (12): 2131-2135.

7. Cohen E, Spierer O. Dry eye post-laser-assisted in situ keratomileusis: major review and latest updates. J Ophthalmol. 2018; 2018: 4903831.

8. Quinto GG, Camacho W, Behrens A. Postrefractive surgery dry eye. Curr Opin Ophthalmol. 2008; 19 (4): 335-341.

9. Huh D, Kay KM, Kim WJ. Superficial punctate keratopathy after laser in situ keratomileusis. J Refract Surg. 2004; 20 (6): 835-836.

10. Konomi K, Chen LL, Tarko RS, Scally A, Schaumberg DA, Azar D et al. Preoperative characteristics and a potential mechanism of chronic dry eye after LASIK. Invest Ophthalmol Vis Sci. 2008; 49 (1): 168-174.

11. Salomão MQ, Ambrósio R Jr, Wilson SE. Dry eye associated with laser in situ keratomileusis: Mechanical microkeratome versus femtosecond laser. J Cataract Refract Surg. 2009; 35 (10): 1756-1760.

12. Contreras-Ruiz L, Ryan DS, Sia RK, Bower KS, Dartt DA, Masli S. Polymorphism in THBS1 gene is associated with post-refractive surgery chronic ocular surface inflammation. Ophthalmology. 2014; 121 (7): 1389-1397.

13. De Paiva CS, Chen Z, Koch DD, Hamill MB, Manuel FK, Hassan SS et al. The incidence and risk factors for developing dry eye after myopic LASIK. Am J Ophthalmol. 2006; 141 (3): 438-445.

14. Murakami Y, Manche EE. Prospective, randomized comparison of self-reported postoperative dry eye and visual fluctuation in LASIK and photorefractive keratectomy. Ophthalmology. 2012; 119 (11): 2220-2224.

15. Nassaralla BA, McLeod SD, Nassaralla JJ Jr. Effect of myopic LASIK on human corneal sensitivity. Ophthalmology. 2003; 110 (3): 497-502.

16. Hu L, Xie W, Liu J, Zhou Y, Zhou Q, Yu Y et al. Tear menisci and corneal subbasal nerve density in patients after laser in situ keratomileusis. Eye Contact Lens. 2015; 41 (1): 51-57.

17. Tao A, Shen M, Wang J, Chen Q, Lu F. Upper and lower tear menisci after laser in situ keratomileusis. Eye Contact Lens. 2010; 36 (2): 81-85.

18. Hammond MD, Madigan WP Jr, Bower KS. Refractive surgery in the United States Army, 2000-2003. Ophthalmology. 2005; 112 (2): 184-190

19. Battat L, Macri A, Dursun D, Pflugfelder SC. Effects of laser in situ keratomileusis on tear production, clearance, and the ocular surface. Ophthalmology. 2001; 108 (7): 1230-1235. 